\title{
String Theory: An Overview
}

\author{
J. Louis ${ }^{1,2}$, T. Mohaupt ${ }^{3}$, and S. Theisen ${ }^{4}$
}

${ }^{1}$ II. Institut für Theoretische Physik, Universität Hamburg, 22761 Hamburg, Germany

2 Zentrum für Mathematische Physik, Universität Hamburg, 20146 Hamburg, Germany jan.louis@desy.de

3 Theoretical Physics Division, Department of Mathematical Sciences, University of Liverpool, Peach Street, Liverpool L69 7ZL, U.K. Thomas. Mohaupt@liverpool.ac.uk

${ }^{4}$ Max-Planck-Institut für Gravitationsphysik, Albert-Einstein-Institut, Am Mühlenberg 1, 14476 Golm, Germany theisen@aei.mpg.de

\section{Introduction}

String theory is not, in contrast to general relativity and quantum field theory, a theory in the strict sense. There is, e.g., no axiomatic formulation and there is no set of defining equations of motion. Instead there is a set of rules which have been developed over the years. They have led to rather spectacular results and have passed all conceivable consistency checks. As has become clear, string theory is more than a theory of strings. This is most apparent through the rôle played by D-branes. They are additional extended dynamical objects whose existence within the theory can be inferred from a variety of arguments. D-branes and other types of p-branes (p-dimensional membranes) are essential for the web of non-perturbative dualities between the known perturbative string theories. Since it is not clear whether strings will remain the fundamental degrees of freedom in the final form of the theory, the term M-theory is frequently used instead of non-perturbative string theory.

However, both notions are programmatic, as the underlying dynamical principle and, closely related to this, the symmetries of string theory have not yet been found. It would thus be more appropriate to speak about a 'theory under construction'; nevertheless, following common usage, we will always speak of string theory or M-theory, the later being understood as the working title for the non-perturbative completion of string theory. At the moment it is not possible to present a well-rounded-off view of string theory. All this non-technical overview is able to accomplish is to recall some of the successes of the theory, mention some of the current activities and some of the open challenges. 


\section{Beyond the Standard Model}

String theory is a proposal for a unifying framework of high energy physics. At currently achievable accelerator energies of $\mathcal{O}(1 \mathrm{TeV})$ or, equivalently, at distance scales $>10^{-19} \mathrm{~m}$, the standard model (SM) of Particle Physics (amended with appropriate neutrino masses) provides a successful and predictive theoretical description. It is based on the mathematical framework of a local quantum gauge field theory with gauge group $S U(3) \times S U(2) \times U(1)$. Nevertheless it is believed that the SM is merely a low-energy effective description of a more fundamental theory. There are several reasons for this belief.

(1) The standard model has many free parameters (coupling constants, mixing angles, etc.) which have to be fixed by experiments. They could, a priori, take any value (within a given range such that the effective description is still valid and that perturbative calculations, on which the comparisons with experiment are based, are justified). In addition there is no explanation of the particle spectrum and its symmetries; they are also experimental input, the only theoretical restriction being the requirement of cancellation of gauge anomalies.

(2) The standard model does not include the gravitational interactions. While this can be safely ignored at laboratory energies, there is nevertheless an energy range where gravity competes with the gauge interactions. This is a consequence of the fact that the gravitational coupling constant $G_{N}$ has dimension of (length) ${ }^{2}$. One should therefore consider the dimensionless quantity $G_{N} E^{2}$, where $E$ is the energy scale of the experiment. With $G_{N} \sim M_{\mathrm{Pl}}^{-2}$ this means that the gravitational interaction becomes large at energies comparable with the Planck scale. It is in this regime that a quantum theory of gravity is needed, provided that we assume that gravity is a fundamental interaction and not an effective one (which would not be quantized).

(3) In the standard model space-time is non-dynamical and smooth. The number of dimensions and the geometry (four-dimensional Minkowski spacetime) are fixed and the back-reaction on the geometry is neglected. However, at very high energies this is no longer appropriate; for instance, if a mass $m$ is squeezed into a volume of a size smaller than its Schwarzschild radius $r_{s} \sim l_{p}^{2} \mathrm{~m}$, then we expect that it will collapse into a black hole.

These points make it highly desirable to have a unified quantum theory of all interactions. Here 'unification' can be understood in two ways. The broader, conceptual meaning of unification is to have a consistent framework which includes both quantum theory and gravity. The predominant belief among particle physicists is that this mainly requires to 'quantize gravity', i.e., to reformulate Einstein's theory of gravity as a quantum theory. However, one should bear in mind that 'quantization' is only an, albeit successful, formal device for formulating quantum theories. It is quite plausible that the introduction of a dynamical space-time requires significant modifications of 
quantum theory as well. We will briefly comment on the stringy perspective on this below.

The 'conceptual' type of unification is not only aesthetically appealing, but also mandatory if we want to address the physics of the early universe and of black holes in a consistent way. The second, more narrow, meaning of unification is that, qualitatively speaking, all forces in nature are manifestations of one single force (as, e.g., in the Kaluza-Klein scenario). The popular though somewhat over-ambitious terms 'theory of everything' or 'Weltformel' have been coined to illustrate this idea. More concretely, one can put forward the working hypothesis that there is a symmetry principle, such that (i) the coupling constants of all interactions can be expressed in terms of one fundamental coupling constant, and such that (ii) all particles organize into irreducible representations. While this idea is aesthetically pleasing, it is by no means a necessary requirement for having a consistent fundamental theory of nature. However, the idea of unification (in the narrow sense) has great heuristic value, as it has stimulated the formulation of interesting theories.

While the unification of the non-gravitational interactions within the framework of quantum gauge theories does not meet fundamental problems, this changes once one attempts to include gravity. One way to see this is due to the perturbative non-renormalizability of the gravitational interaction 1

In this context, the main differences between the gravitational and the renormalizable Yang-Mills gauge interactions are (1) the graviton has spin two while Yang-Mills gauge bosons have spin one; (2) the gravitational coupling constant has negative mass-dimension while the gauge coupling constants are dimensionless. Difference (2) renders the theory of gravity, based on the Einstein-Hilbert action, perturbatively non-renormalizable: the UV infinities in Feynman diagrams cannot be absorbed by a finite number of local counter-terms. The cure for the Fermi theory of weak interactions, that is pulling the four-fermion interaction apart by inserting a propagator line of (massive) gauge bosons, does not work for the theory of gravity with its infinity of interaction vertices. However, if one expands the individual lines of a Feynman diagram into tubes or strips, thus replacing the world-lines by world-sheets of closed or open strings, one solves, in one go, the problem with UV infinities and replaces the infinitely many interaction vertices by a finite number of basic three-point interactions (cf. Fig. 1).

In addition, all elementary particles, gauge fields and matter fields, correspond to vibration modes of the string, which is the only fundamental object (in contrast to a quantum field for each particle species as, e.g., in grand unification models). Since every consistent quantum theory of strings necessarily contains a massless spin two particle (which has the kinematical and dynamical properties of a graviton), it automatically includes gravity. Therefore string theory is a unified theory in both meanings of the word.

\footnotetext{
${ }^{1}$ We will not discuss conceptual problems but refer instead to the contributions on quantum gravity in this book.
} 


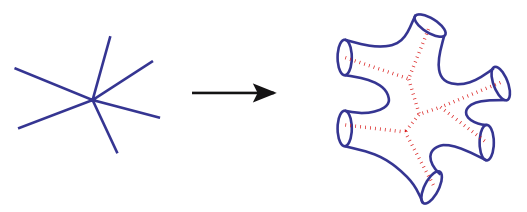

Fig. 1. 'Thickening' a field theory interaction vertex

While unification in the narrow sense is manifest in the formalism, the conceptual unification of quantum theory and gravity has not yet been achieved in a satisfactory way. The reason for this is that the formalism of 'perturbative string theory', to be reviewed in Sects. 2 and 3. only gives a set of rules for computing on-shell scattering amplitudes in an on-shell background. While the UV finiteness of string amplitudes, which has been made highly plausible (though not been proved rigorously), is clearly relevant for the conceptual unification, it mainly takes care of a technical aspect. Given that the amplitudes are indeed finite, string theory provides only a perturbative theory of quantum gravity. Note, however, that this allows to do more than just to compute graviton scattering in a fixed background. It also allows to compute an effective action, which encodes quantum corrections to the Einstein-Hilbert term. This in turn has implications for black hole physics, which will be the subject of Sect. 8. At this point we just emphasize that conceptual issues of quantum gravity, such as black hole entropy, have been addressed successfully in string theory. This said, it must also be stressed that the range of conceptual points which can be addressed today is quite limited. The main reason is that as a starting point one always has to specify a reference background space-time. We will come back to this when discussing open questions in Sect. 10.

Next, let us briefly come back to the question whether the conceptual unification of quantum theory and gravity mainly requires to 'quantize gravity', or whether both quantum theory and gravity need to be modified in a more drastic way. In perturbative string theory quantization is applied in the same pragmatic spirit as in theoretical particle physics. Actually, the approach is at first glance a bit more naive, as one quantizes the relativistic string and thus does quantum mechanics rather than quantum field theory. The fact that this procedure results in a consistent perturbative theory of quantum gravity is a surprising discovery, and the deeper reason behind this remains to be understood. Heuristically, the improved UV behaviour can be understood in terms of the 'thickening' of propagators and vertices, which we mentioned above. As a consequence, classical physics is modified in two ways, not only by quantum corrections, but also by stringy corrections related to the finite size of strings. As we will see later, the string length replaces the Planck length as the fundamental scale (at least in perturbative string theory), while there are also transformations ('dualities') in the theory, which mutually exchange quantum corrections and stringy corrections. While the deeper implications of these observations remain to be explored, it indicates that the full theory does more 
than just 'quantize gravity'. The relation between string theory and quantum field theory is more complicated than suggested by the naive picture of 'thickening Feynman graphs'. While programmatically string theory intends to supersede quantum field theory, in its current state it is deeply entangled with it. The first point which makes this obvious is the rôle of two-dimensional conformal field theories, which we will elaborate on in Sects. 3 and 4 . While string scattering amplitudes are finite, the two-dimensional field theories used in the Polyakov approach are just renormalizable. Therefore the concept of renormalization still plays a rôle. The second point, to be discussed in Sect. 7, is the AdS/CFT correspondence, which claims that string theory in certain backgrounds is equivalent to specific quantum field theories. Here, and in related proposals such as the so-called 'M(atrix) theory', one even contemplates to define string theory in terms of quantum field theory. Let us further note a trend shared by string theory and quantum field theory, namely the importance of effective field theories. Here again renormalization (understood in a Wilsonian spirit) plays an important rôle. Of course, the concept of string effective field theories is by itself consistent with the idea that string theory supersedes quantum field theory. However, the two previous examples show that in its present state string theory has a more complicated relationship with quantum field theory. The only systematic approach to go beyond local quantum field theory is string field theory, which aims to be a full-fledged quantum field theory of extended objects. Unfortunately, string field theory has proved to be complicated that progress was very slow. Moreover, it is not clear how the non-perturbative dualities, to be discussed in Sect. 6, which nowadays hold a central position in our understanding of string theory, fit together with string field theory.

The optimists hope, of course, that all the exciting observations made during the last years will ultimately condense into a new principle, which supersedes and conceptually unifies quantum field theory and gravity. But so far only some clues have been found, while the 'big picture' is still far from clear.

\section{The Free String}

The dynamics of the bosonic string in $d$-dimensional Minkowski space-time is governed by the Nambu-Goto action

$$
S_{\mathrm{NG}}=\frac{1}{2 \pi \alpha^{\prime}} \int_{\Sigma} d \sigma d \tau \sqrt{|\operatorname{det} G(X)|} .
$$

The world-sheet $\Sigma$ which is swept out by the string is parametrized by $\sigma^{\alpha}=(\sigma, \tau)$. The integral is the area of $\Sigma$ measured with the induced metric $G_{\alpha \beta}=\partial_{\alpha} X^{\mu} \partial_{\beta} X^{\nu} \eta_{\mu \nu} . X^{\mu}(\sigma, \tau): \Sigma \hookrightarrow M$ is the embedding of $\Sigma$ into the $d$-dimensional space-time with Minkowski-metric $\eta_{\mu \nu}, \mu, \nu=0, \ldots, d-1$. $T=\frac{1}{2 \pi \alpha^{\prime}}$ is the string tension. $l_{\mathrm{s}}=\sqrt{\alpha^{\prime}}$ is the string scale, a length scale characteristic for string theory. It replaces the Planck length $l_{\mathrm{p}}$ as the fundamental length scale. 


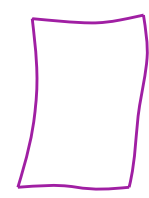

(a)

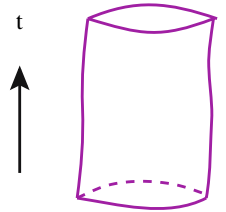

(b)

Fig. 2. World-sheet of a free (a) open and (b) closed string

$S_{\mathrm{NG}}$ is the direct generalization of the action of a point-like (i.e. zerodimensional) relativistic particle to one-dimensional strings. The world-sheet of a freely propagating open and closed string has the topology of a strip and cylinder, respectively (Fig. 2). For the latter, $X^{\mu}(\sigma, \tau)$ is periodic (in $\sigma$ ) on the cylinder.

For the open string one can impose either Dirichlet (D) or Neumann (N) boundary conditions for each of the $d$ fields $X^{\mu}$ at each of the two ends of the string. The physical meaning of Neumann boundary conditions is that space-time momentum does not flow off the ends of the string. With Dirichlet boundary conditions the position of the end of the string is fixed while spacetime momentum flows off. $d$-dimensional Poincaré invariance demands that the total space-time momentum is conserved. This means that momentum must be absorbed by other dynamical objects. These objects, on which open strings end, are called Dirichlet branes, or D-branes, for short (cf. Fig. 3). If $p$ of the spatial components of $X^{\mu}$ at one end of the string have Neumann boundary conditions and the remaining $d-p-1$ components have Dirichlet boundary conditions, this string ends on a D p-brane. A D0-brane is also called D-particle and a D1-brane is called D-string. Fundamental strings (F-strings) and D-string are quite different objects. One difference is that an open F-string must end on a D-brane (but not vice versa). Other differences will be discussed below.

Propagation of the particles which correspond to the excitations of the open string is restricted to the world-volume of the D-brane while excitations of the closed string propagate in the full $d$-dimensional space-time.

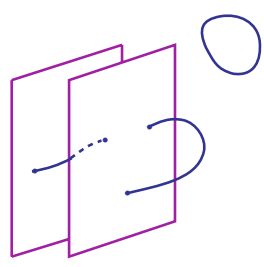

Fig. 3. Open strings end on D-branes; closed strings explore the $d$-dimensional space-time 
Quantization of string theory simplifies if one uses the Polyakov action $S_{\mathrm{P}}$ which is classically equivalent to the Nambu-Goto action:

$$
S_{\mathrm{P}}=-\frac{1}{4 \pi \alpha^{\prime}} \int_{\Sigma} d \sigma d \tau \sqrt{|\operatorname{det} h|} h^{\alpha \beta} \partial_{\alpha} X^{\mu} \partial_{\beta} X^{\nu} \eta_{\mu \nu} .
$$

$S_{\mathrm{P}}$ is the action of $d$ scalar fields $X^{\mu}(\sigma, \tau)$ which are coupled to twodimensional gravity with metric $h_{\alpha \beta}(\sigma, \tau) . S_{\mathrm{P}}$ is invariant under (i) global space-time Poincaré transformations $X^{\mu} \rightarrow a^{\mu}{ }_{\nu} X^{\nu}+b^{\mu}, a^{\mu}{ }_{\rho} a^{\nu}{ }_{\sigma} \eta_{\mu \nu}=\eta_{\rho \sigma}$; (ii) local reparametrizations of the world-sheet $\sigma^{\alpha} \rightarrow \tilde{\sigma}^{\alpha}(\sigma, \tau)$; and (iii) under local Weyl-rescalings of the metric $h_{\alpha \beta} \rightarrow \Omega^{2}(\sigma, \tau) h_{\alpha \beta}$. Local Weyl invariance implies tracelessness of the energy-momentum tensor $T_{\alpha \beta}=-\frac{4 \pi}{\sqrt{|\operatorname{det} h|}} \frac{\delta}{\delta h^{\alpha \beta}} S_{p}$ of the world-sheet field theory, i.e. $h^{\alpha \beta} T_{\alpha \beta}=0$. Reparametrization invariance can be used to go to conformal gauge 2 where $h_{\alpha \beta}=e^{2 \varphi(\sigma, \tau)} \eta_{\alpha \beta}$. In the classical theory the Weyl degree of freedom $\varphi$ decouples. Violation of the local Weyl invariance in the quantized theory is signalled by a conformal anomaly. It is measured by the central charge of the Virasoro algebra, the algebra of constraints $\left(T_{\alpha \beta}=0\right)$ in the quantized theory.

In the $(1,1)$ supersymmetric version of the Polyakov action, every bosonic field $X^{\mu}$ and its two Majorana-Weyl superpartners $\psi_{ \pm}^{\mu}$ of positive and negative chirality are coupled to two-dimensional world-sheet supergravity $\left(h_{\alpha \beta}, \chi_{\alpha}^{ \pm}\right)$. In the classical theory, in addition to the metric degrees of freedom also those of the two world-sheet gravitini $\chi_{\alpha}^{ \pm}$are unphysical. This is a consequence of two-dimensional local world-sheet supersymmetry.

The fermions $\psi_{ \pm}^{\mu}$ on the world-sheet of the closed string can be periodic (Ramond) or anti-periodic (Neveu-Schwarz), where the periodicity condition can be chosen independently for each chirality. This leads to four different sectors of the closed string theory 3 Excitations in the (NS,NS) and the (R,R) sectors are space-time bosons while excitations in the two mixed sectors, $(\mathrm{R}, \mathrm{NS})$ and (NS,R), are space-time fermions. For the open string the boundary conditions couple the two chiralities to each other. This leads to two sectors: the NS sector with space-time bosons and the R sector with space-time fermions.

Quantization of string theory in Minkowski space-time is only possible in the critical dimension $d_{\text {crit }}$, unless one is willing to accept that the quantum theory of strings contains an additional degree of freedom, the Liouville mode.

${ }^{2}$ Going to conformal gauge does not fix the reparametrization invariance completely. The remaining transformations are (in Euclidean signature on $\Sigma$ ) conformal transformations and the two-dimensional field theory on $\Sigma$ in conformal gauge is a so-called 'conformal field theory'.

3 Classically, one could define different theories by taking any subset of the possible boundary definitions. However, the quantum theory includes multiply connected world-sheets, and the theory must be invariant under the so-called 'modular transformations', to be discussed below. This in turn implies that all combinations of boundary conditions have to be included, and thus each type of boundary condition defines a sector of the quantum theory. 
The status of Liouville string theory, also known as non-critical string theory, is not completely understood. Throughout this chapter we will fix the number of space-time dimensions to be $d_{\text {crit }}$. The critical dimension is $d_{\text {crit }}=26$ for the bosonic string and $d_{\text {crit }}=10$ for the fermionic string 4 One obtains a positivedefinite Hilbert space (no-ghost-theorem) and space-time Poincaré invariance as well as (super)Weyl invariance on the world-sheet are anomaly free. In the covariant BRST quantization the gauge fixing of the local symmetries leads to ghost fields, the reparametrization ghosts $(b, c)$, and their superpartners $(\beta, \gamma)$. In the critical dimension their contribution to the conformal anomaly is compensated by $X^{\mu}$ and $\psi^{\mu}$.

The resulting spectrum of the theory contains a finite number of massless and infinitely many massive excitations with mass ${ }^{2}=\frac{n}{2 \alpha^{\prime}}$ with $n \in \mathbb{N}$. Among the states there are also tachyons with negative mass ${ }^{2}$. They imply an instability of the vacuum. This is unavoidable in the bosonic string. However, the spectrum of the fermionic string must be truncated by an additional projection, the Gliozzi-Scherk-Olive (GSO) projection. This projection can be chosen such that the tachyon is projected out and the remaining spectrum is space-time supersymmetric. The GSO projection is necessary and can be understood as a consistency condition (modular invariance, locality of the CFT operator products) which must be imposed on the quantum mechanical scattering amplitudes, to be discussed in the next section. In a theory with only closed strings the spectrum has $\mathcal{N}=2$ space-time supersymmetry. Two possible, inequivalent GSO projections lead to the non-chiral type IIA and to the chiral type IIB theory. Their massless spectra are those of ten-dimensional type IIA and type IIB supergravity, respectively.

The spectrum of type I theory with both open and closed strings is $\mathcal{N}=1$ supersymmetric. Its massless spectrum is that of supersymmetric Yang-Mills theory, coupled to supergravity. The degrees of freedom of the Yang-Mills theory are excitations of the open string. The two ends of the open string carry charges in the fundamental representation of the gauge group (ChanPaton factors) such that the open string has the quantum numbers of a gauge boson. The supergravity degrees of freedom are, as in the type II theories, the massless excitations of the closed string. Consistency requires the gauge group to be $S O(32)$. Only in this case gauge and gravitational anomalies vanish.

Type I and type II theories are also called superstring theories. In addition to the type I theory, there are two further string theories with $\mathcal{N}=1$ space-time supersymmetry. These are the heterotic $E_{8} \times E_{8}$ and $S O(32)$ theories. These theories have, like the type II theories, only closed strings. In contrast to the local $(1,1)$ world-sheet supersymmetry of the superstring,

4 Strictly speaking, one does not need to fix the number of space-time dimensions, but the number of degrees of freedom, as measured by the central charge of the world-sheet conformal field theory, which must be $c=26$ and $c=10$ in order to cancel the contribution from the reparametrization ghosts. The surplus degrees of freedom need not have the interpretation of string coordinates along extra dimensions. 
heterotic theories have local $(1,0)$ supersymmetry. The superpartner of $X^{\mu}$ is a single Majorana-Weyl fermion $\psi_{+}^{\mu}$. Absence of gravitational anomalies on the world-sheet requires, in the fermionic formulation of the theory, 32 additional Majorana-Weyl fermions $\lambda_{-}^{a}, a=1, \ldots, 32$. In the bosonic formulation these 32 fermions are replaced by 16 periodic chiral scalars $\Phi^{I}(\tau+\sigma), I=1, \ldots, 16$ which are the coordinates of a 16-dimensional torus. Modular invariance restricts the allowed tori to those which are generated by a 16-dimensional self-dual even lattice $\Lambda$ via $T^{16}=\mathbb{R}^{16} / \Lambda$. There are precisely two such lattices which lead to the two allowed gauge groups $E_{8} \times E_{8}$ and $S O(32)$. The massless spectra of the two heterotic theories are again those of supersymmetric Yang-Mills theory, coupled to supergravity, now with gauge group $E_{8} \times E_{8}$ or $S O(32)$.

\section{The Interacting String}

The discussion in Sect. 3 was based on the free string theory. Interactions are introduced through the inclusion of topologically non-trivial world-sheets. Figure 4 shows the decay of a closed string into two closed strings, while Fig. 5 shows the joining of two open strings into a closed string.

The strength of the interaction is controlled by the value of the dimensionless string coupling constant $g$, which is dynamically determined through the background value (vacuum expectation value) $\Phi_{0}$ of the dilaton $\Phi, g=e^{\Phi_{0}}$. The dilaton, as the graviton, is part of the massless spectrum of every string theory. Different values of $g$ do not correspond to different theories but they parametrize ground states 5 of a given theory. The coupling constants of the different string theories are, however, a priori independent.

In string theory, the quantum field theoretical computation of scattering amplitudes by summation over Feynman diagrams is replaced by the summation over world-sheets of different topologies (cf. Fig. 6). Which topologies are

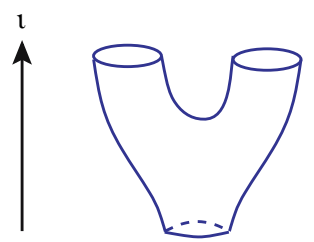

Fig. 4. Decay of a closed string into two closed strings

\footnotetext{
${ }^{5}$ By a (perturbative) string ground state ('string vacuum') we mean a conformal field theory with the correct properties, i.e. the correct central charge, modular invariant partition function, etc. A geometric realization can be provided by specific background configurations of the massless fields. In this section we choose $G_{\mu \nu}(X)=\eta_{\mu \nu}, \Phi=\Phi_{0}$ with all others set to zero. More general backgrounds will be mentioned in later sections.
} 


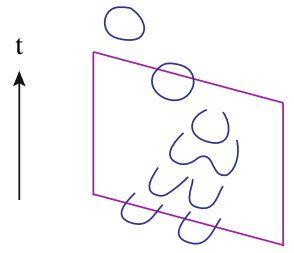

(a)

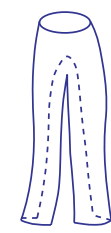

(b)

Fig. 5. Two open strings join to a closed string (a) in a space-time diagram and (b) in a world-sheet diagram

allowed depends on the string theory. In particular, in type I both orientable and non-orientable world-sheets must be summed over while the world-sheets of the four other theories must be orientable.

Scattering amplitudes $\mathcal{A}$ can be computed in perturbation theory. $\mathcal{A}$ is expanded in a power series in the string coupling $g$

$$
\mathcal{A}=\sum_{n} g^{n} \mathcal{A}^{(n)}
$$

where each term $\mathcal{A}^{(n)}$ is computed separately. The validity of perturbation theory requires $g \ll 1$. The power of $g$ with which a given world-sheet contributes is the negative of its Euler number, i.e. it is determined by its topology. The scattering amplitudes $\mathcal{A}^{(n)}$ of physical states are correlation functions of BRST-invariant vertex operators of the quantum field theory on $\Sigma$ as specified by the Polyakov action. In the path-integral formulation one has to sum over all metrics $h_{\alpha \beta}$ on $\Sigma$ and over all embeddings $X^{\mu}$ of $\Sigma$ in space-time $M$. The computation of scattering amplitudes is most easily done with the methods of conformal field theory. Using the local symmetries on $\Sigma$ one goes to (super) conformal gauge and the infinite dimensional integration over $h_{\alpha \beta}\left(\chi_{\alpha}^{ \pm}\right)$is reduced to the finite dimensional integration over the (super) moduli of $\Sigma$ and the integration over the Faddeev-Popov ghosts. For closed strings, requiring invariance of the amplitudes under those reparametrizations which are not continuously connected to the identity transformation (modular invariance) restricts the range of integration of the modular parameters to a fundamental region. Requiring modular invariance for the one-loop amplitudes guarantees

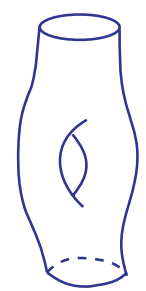

Fig. 6. One-loop quantum correction to the propagation of a closed string 
the anomaly freedom of the space-time spectrum. It also is equivalent to imposing the GSO projection on the spectrum. For open strings one has to impose, in addition, a tadpole cancellation condition for the (R,R)-fields.

For external momenta which are small compared to the characteristic scale $l_{s}^{-1}=1 / \sqrt{\alpha^{\prime}}$, the scattering amplitudes coincide with those of an effective field theory. Expanding the amplitudes in powers of $\alpha^{\prime}$ corresponds to an expansion in powers of derivatives in the effective field theory. In leading order the lowenergy effective action of each of the five string theories coincides with the action of the appropriate classical supergravity theory. The relations between the coupling constants of supergravity (the ten-dimensional Newton's constant $G_{\mathrm{N}}^{(10)}$ and the ten-dimensional Yang-Mills coupling $\left.g_{\mathrm{YM}}\right)$ and $g$ and $l_{s}$ are $G_{\mathrm{N}}^{(10)} \sim g^{2} l_{s}^{8}, g_{\mathrm{YM}}^{2} \sim g^{2} l_{s}^{6}$ (heterotic), and $g_{\mathrm{YM}}^{2} \sim g l_{s}^{6}$ (type I). (Computable) corrections in $\alpha^{\prime}$ result, e.g., in a modification of the Einstein-Hilbert action by terms which contain higher powers of the Riemann tensor.

The finite extent of the string becomes relevant for external momenta $\mathcal{O}\left(1 / \sqrt{\alpha^{\prime}}\right)$ where deviations from quantum field theory become noticeable. For instance, in contrast to quantum field theory, string scattering amplitudes are UV finite. Heuristically this can be understood as follows: the point-like interaction vertices of QFT are now smeared. Perturbative finiteness of string theory 6 is a consequence of modular invariance which restricts the analogue of the Schwinger proper time-integral to a so-called 'fundamental region'. (Modular invariance is a property of string theory and requires, as a necessary condition, the excistence of an infinite number of excitations.) Within the framework of string theory one can compute perturbative corrections to the gravitational interaction. It is in this sense that string theory (in its supersymmetric version) is an UV finite and unitary perturbative quantum theory of gravity.

\section{Compactification}

So far the discussion was restricted to string theories in a given $d_{\text {crit }}{ }^{-}$ dimensional Minkowski space-time with metric $\eta_{\mu \nu}$. However, it is possible to formulate string theory in topologically and metrically non-trivial spacetimes where, e.g., only $d$ dimensions are infinitely extended and the remaining $d_{\text {int }}=d_{\text {crit }}-d$ are curled up and compact. One possible realization of such a compactification starts with a direct product Ansatz $M^{d} \times K^{\text {int }}$ for the tendimensional space-time. Here $M^{d}$ is $d$-dimensional Minkowski space and $K^{\text {int }}$

\footnotetext{
${ }^{6}$ A complete all-order proof of perturbative finiteness has not been worked out yet. While there are technical difficulties related to gauge fixing of the supermoduli at higher loops, there is no apparent obstruction to extending the existing finiteness results to all loops. (These difficulties seem to be absent in Berkovits' covariant and manifestly space-time supersymmetric quantization of the superstring.) This is different from the situation in maximally extended supergravity, where counterterms are possible, and, hence, to be expected, at higher loop level.
} 
a $d_{\text {int }}$-dimensional compact manifold. In the Polyakov action this corresponds to replacing $\eta_{\mu \nu}$ by the metric $g_{\mu \nu}(X)$ of the product space. This leads to a two-dimensional non-linear $\sigma$-model with target space $M^{d} \times K^{\text {int }}$. Consistency of the compactification requires a conformally invariant $\sigma$-model and thus implies strong restrictions on $K^{\text {int }}$. The resulting $d$-dimensional theory depends on the geometry and topology of the compact manifold. For instance $d$-dimensional supersymmetric theories require that the manifold $K^{10-d}$ admits Killing spinors.

The simplest consistent compactification of a closed string is on a circle $S^{1}$ with radius $R$. One requires $X(\sigma+2 \pi, \tau)=X(\sigma, \tau)+2 \pi w R, w \in \mathbb{Z}$, for one of the coordinates. This leads to additional massless and massive states: On the one hand, the Kaluza-Klein excitations with mass ${ }^{2}=(n / R)^{2}, n \in \mathbb{Z}$ which decouple for $R \rightarrow 0$ and, on the other hand, winding states with mass $^{2}=\left(w R / l_{s}^{2}\right)^{2}$, which become massless for $R \rightarrow 0$. These winding states are characteristic for string theory and are not present in compactified field theories. They lead to a symmetry of the spectrum and the scattering amplitudes of the bosonic string under the $T$-duality transformation $R \rightarrow l_{s}^{2} / R, g \rightarrow g l_{s} / R$ under which Kaluza-Klein and winding states are exchanged. In other words geometrically different compactifications correspond to physically identically ground states of string theory. This symmetry implies to regard $l_{s}$ as minimal length: Compactifications on a large circle is indistinguishable form compactification on a small circle. In both cases the limits $R \rightarrow \infty$ and $R \rightarrow 0$, respectively, lead to a continuum of massless states which is interpreted as the decompactification of an additional dimension. The compactification on $S^{1}$ leads to an additional free parameter, the radius $R$ of the circle. Similar to the string coupling $g$ it can be interpreted as the vacuum expectation value of a massless scalar field (modulus); e.g. $G_{25,25}=R^{2}$ for the bosonic string compactified on a circle in the $X^{25}$ direction.

The ground states of the compactified theory are restricted via $T$-duality to either one of the two fundamental regions $R \in\left[l_{s}, \infty\right)$ or $R \in\left(0, l_{s},\right]$. While $T$-duality is a symmetry of the bosonic string this is not the case for type II strings: $T$-duality transforms type IIA theory on $S_{R}^{1}$ to type IIB theory on $S_{l_{s}^{2} / R}^{1}$.

A simple generalization of a compactification on a circle is the compactification on a $d_{\text {int-dimensional torus }} T^{d_{\text {int }}}$. Here $T$-duality is a non-Abelian discrete symmetry on the parameter space (moduli space) of the compactification whose local coordinates are, among others, the components of the metric on $T^{d_{\text {int }}}$.

Of physical interest is the case $d=4$. For type II theories compactification on $T^{6}$ leads, at the level of the low-energy effective action, to $\mathcal{N}=8$ supergravity and for the type I and the two heterotic theories to $\mathcal{N}=4$ Super-Yang-Mills (SYM) theory coupled to $\mathcal{N}=4$ supergravity. $\mathcal{N}=1(2)$ supergravity is obtained by compactification of the heterotic (type II) string 
on six-dimensional Calabi-Yau manifolds 7 The large number of topologically different Calabi-Yau manifolds leads to many different four-dimensional theories which differ in their spectra of string excitations. This, in turn, leads to different low-energy effective actions which differ from each other in gauge group, spectrum of massless particles, and interactions. Size and shape of the Calabi-Yau manifold are parametrized by the (perturbatively) undetermined vacuum expectation values of neutral (under the gauge group) scalar fields, the moduli fields.

Discrete symmetries which act on the moduli space of a given compactification and which are exact in every order of string perturbation theory are called T-duality. Mirror symmetry of Calabi-Yau compactifications is a non-trivial example of a T-duality. It states that compactifications on a pair of topologically different Calabi-Yau manifolds, a so-called 'mirror pair', are completely equivalent and undistinguishable. This, as already the simple example of the compactification on a circle, demonstrates that strings probe the geometry of a manifolds quite differently than point particle probes. One therefore speaks of 'string geometry'.

In the language of conformal field theory, compactification of a superstring theory means that one replaces $(10-d)$ of the free superfields $\left(X^{i}, \psi^{i}\right)$ by a superconformal non-linear sigma-model with target space $K^{\text {int }}$ and the same central charge $c^{\text {int }}=3 d_{\text {int }} / 2$. More generally one can take an 'internal' superconformal field theory of the same central charge as long as it satisfies consistency conditions such as modular invariance. Such a theory has, in general, no formulation as a sigma-model and does thus not admit a geometric interpretation. An analogous discussion also holds for heterotic theories where the contributions of the additional fields $\lambda^{a}$ or $\Phi^{I}$ have to be taken into account.

More general compactifications than the one discussed so far are not only specified by the metric on $K^{\text {int }}$ but by additional non-trivial background values of other massless bosonic fields. For example, a consistent compactification of type IIB on $A d S_{5} \times S^{5}$ needs a non-trivial background value for the self-dual five-form field strength $F_{5}$ which provides the necessary vacuum energy density to balance the curvature of each factor. As for Calabi-Yau manifolds this compactification is an exact conformal field theory and it plays a prominent rôle in the AdS/CFT correspondence which we discuss in Sect. 7 .

For more general compactifications with background fields, their backreaction on the geometry demands that one gives up the (geometric) direct product structure of the Ansatz and replaces it by a warped product where the metric of the infinitely extended space-time depends on an overall scale factor - the warp factor - which can be a non-trivial function of the coordinates of the compact space. Examples are compactifications where the Calabi-Yau manifold is replaced by a manifold with $S U(3)$-structure (rather than $S U(3)$ holonomy). Such generalized compactifications arise when localized sources

\footnotetext{
${ }^{7}$ Calabi-Yau manifolds are compact Kähler manifolds with $S U(3)$ holonomy.
} 
for the background fields (D-branes, orientifold planes) and/or background fluxes are present, i.e. non-vanishing VEVs for the (R,R) and (NS,NS) antisymmetric tensor fields (see also Sect. 6).

\section{Duality and M-Theory}

So far we have only discussed the perturbative quantization of strings which propagate through a fixed classical background space-time. A complete theory of quantum gravity should, however, dynamically generate the background space-time. At this time, string theory has not yet achieved this, but there has been recent progress within the AdS/CFT correspondence.

The main problem in taking space-time to be dynamical ab initio is that a non-perturbative formulation of the theory does not yet exists. This situation is quite unusual. One often encounters that a theory is, at least in principle, known but in order to compute quantities of interest one must develop perturbative methods which allow approximate computations. In string theory the situation is quite different: only the perturbation series is known while the fundamental formulation from which it can be derived is still lacking.

One possible way to access the non-perturbative regime is via the duality between weakly and strongly coupled theories, a concept which is well known for supersymmetric quantum field theories. It provides control over the strongly coupled regime of a given theory via perturbative methods applied to the dual theory. The two theories which comprise a duality pair are often very different perturbatively; they might differ, e.g., in their degrees of freedom and their symmetries. The perturbative degrees of freedom of one theory might be solitons, i.e. localized solutions of the classical equations of motion of the weakly coupled dual theory. These solitons are not part of the perturbative spectrum since their masses diverge as the coupling constant $g$ approaches zero. If the solitons become very light and weakly coupled as $g \rightarrow \infty$, they might play the rôle of the elementary degrees of freedom of the dual theory. A duality between a weakly and a strongly coupled theory is called S-duality.

S-duality in string theory is non-perturbative in the power series expansion in the coupling constant $g$, but it is perturbative in the expansion in $l_{s}$. For Tduality the situation is reversed. The non-perturbative nature in the expansion in $l_{s}$ manifests itself, e.g. in mirror symmetry, through the contribution of world-sheet instantons $\sim e^{-R^{2} / l_{s}^{2}}$, where $R$ is the overall size of the CalabiYau manifold. A discrete symmetry which is neither perturbative in $g$ nor in $l_{s}$ is called U-duality.

To prove S-duality (or U-duality) is difficult, since it presupposes a nonpertubative formulation of the theory. However, one can check the duality hypothesis on those solitonic states whose quantum corrections are controllable and whose masses, as functions of the coupling constants, can be exactly determined at weak coupling. For these states an extrapolation to strong 
coupling is allowed and the comparison with perturbative states of the dual theory is then possible. Such Bogomolny-Prasad-Sommerfield (BPS) states are present in field and string theories with extended supersymmetry. They have the distinctive property that they preserve some of the supersymmetries, i.e. they are annihilated by some of the supercharges, the generators of the supersymmetry algebra.

The BPS-spectrum of string theory contains, in particular, the D-branes. In analogy to the coupling of an electrically charged particle to the Maxwell potential $A^{(1)}$, an 'electric' $p$-dimensional $\mathrm{D} p$-brane couples to a $(p+1)$ form potential $C^{(p+1)}$. In addition to the electrically charged branes there are also 'magnetically charged' branes. They are characterized through the field strength $(* H)^{(8-p)}$ which is dual to $H^{(p+2)}=d C^{(p+1)}$. This means that the object which is dual (in the sense of Hodge duality) to an electrically charged $\mathrm{D} p$-brane is a magnetically charged $(6-p)$-dimensional $\mathrm{D}(6-p)$ brane. The potentials $C$ to which branes couple are the massless fields in the $(\mathrm{R}, \mathrm{R})$ sectors of superstring theories.

The (NS,NS) sector of the type II and the heterotic string theories also contains an anti-symmetric tensor field $B_{\mu \nu}$ to which their fundamental string (F1) couples. The dual magnetic object is the five-dimensional NS5-brane. The massless bosonic fields and the D-brane spectra of the different string theories are summarized in Tables 1 and 2 . The massless fermionic fields are determined by space-time supersymmetry.

Branes were first discovered as classical solutions of the effective supergravity theories. The supergravity solutions describe extended objects and contain, in addition to a non-trivial space-time metric and the dilaton, a non-vanishing $(p+2)$-form field strength $H^{(p+2)}$. Subsequently the solutions which couple to $(\mathrm{R}, \mathrm{R})$ fields got their string theoretic interpretation as D-branes, namely the dynamical objects on which open strings end and

Table 1. Bosonic massless fields in type II theories, the closed string sector of type I and in the heterotic theories. $G_{\mu \nu}$ is the space-time metric, $B_{\mu \nu}$ an anti-symmetric tensor field (Kalb-Ramond field), and $\Phi$ the dilaton. $A_{\mu}$ is the vector potential of the gauge groups $E_{8} \times E_{8}$ and $S O(32)$, respectively. $C^{(p)}$ is a $p$-form field with field strength $H^{(p+1)}=d C^{(p)}$. The field strength of $C^{(4)+}$ is self-dual, $H^{(5)}=* H^{(5)}$, and $H^{(0)}$ is a non-propagating 0 -form field strength. (The type I string also has $\mathrm{SO}(32)$ gauge bosons from the open string sector)

\begin{tabular}{lll}
\hline Sektor & $(\mathrm{NS}, \mathrm{NS})$ & $(\mathrm{R}, \mathrm{R})$ \\
\hline Type IIA & $G_{\mu \nu}, B_{\mu \nu}, \Phi$ & $H^{(0)}, C^{(1)}, C^{(3)}$ \\
Type IIB & $G_{\mu \nu}, B_{\mu \nu}, \Phi$ & $C^{(0)}, C^{(2)}, C^{(4)+}$ \\
Type I & $G_{\mu \nu}, \Phi$ & $C^{(2)}$ \\
Heterotic & $G_{\mu \nu}, B_{\mu \nu}, \Phi, A_{\mu}$ & \\
\hline
\end{tabular}


Table 2. D-brane spectra of superstring theories. The $\mathrm{D}(-1)$ brane of type IIB is a D-instanton. The D9 brane in type I is degenerate. It implies that open strings can move freely in the ten-dimensional space-time. All remaining D-branes are in one-to-one correspondence to 'electric' (R,R)-potentials and their 'magnetic' duals. D-branes couple to these potentials as sources. T-duality changes the boundary conditions of open strings, $\mathrm{N} \leftrightarrow \mathrm{D}$. This means that $\mathrm{T}$-duality maps $\mathrm{D} p$-branes to $\mathrm{D}(p \pm 1)$-branes, depending on whether the T-duality direction is along $(-)$ or perpendicular $(+)$ to the world-volume of the brane. Type II and heterotic theories also have a NS5 brane

\begin{tabular}{ll}
\hline D $p$-branes & $p$ \\
\hline type IIA & $0,2,4,6,8$ \\
type IIB & $-1,1357$ \\
type I & 159 \\
\hline
\end{tabular}

to which they transfer space-time momentum. Those which couple to $B_{\mu \nu}$ or its dual are identified with the fundamental string and the NS5 brane, respectively. If one computes the tension (energy density) of the F1 solution (which carries 'electric' $B$-charge) one finds that it is independent of the string coupling constant. The tension for the NS5-brane (which carries 'magnetic' $B$-charge) however, behaves as $\tau_{\mathrm{NS} 5} \sim 1 / g^{2}$ while that of D-branes depends on the string coupling as $\tau_{\mathrm{D}} \sim 1 / \mathrm{g}$. This means that the NS5- and the D-branes are heavy and decouple in the weak coupling limit $g \rightarrow 0$. They are part of the non-perturbative sector of the respective pertubatively defined string theory. At strong coupling, $g \gg 1$, the BPS- $p$-branes become light. In some cases they can be viewed as the fundamental objects of a dual theory which possesses a perturbative expansion in powers of $g_{\text {dual }}=1 / g$.

An example of this in $d=10$ is the S-duality between the heterotic $\mathrm{SO}(32)$ string and the type I string. The coupling constants of these two theories are inverse of each other, and the D-string of type $\mathrm{I}$ is mapped, in the limit of strong coupling, to the fundamental heterotic string.

The type IIB theory in $d=10$ possesses both an F-string and a D-string. The relation between their tensions is $\tau_{\mathrm{F} 1} / \tau_{\mathrm{D} 1}=g$, i.e. at strong coupling the $\mathrm{D}$-string is much lighter than the F-string. The type IIB theory is self-dual under S-duality, i.e. it is invariant under $g \rightarrow 1 / g$ and simultaneous exchange of D- and F-strings and their dual magnetic objects, the D5 and NS5 branes. T-duality relates the type IIB theory with the type IIA theory. T-duality also relates the two heterotic theories with each other.

The type IIA theory has BPS bound states of $n$ D0-branes with mass $m \sim n /\left(g l_{s}\right)$. These states can be interpreted as Kaluza-Klein excitations of an 11-dimensional theory which has been compactified on an $S^{1}$ with radius $R_{11}=g l_{s}$ (cf. the discussion of $S^{1}$ compactification in Sect. 5). In the strong coupling limit $g \rightarrow \infty$, the type IIA theory possesses 11-dimensional Poincaré invariance. At low energies the massless excitations and their 
interactions are described by the unique 11-dimensional supergravity theory. Using $G_{N}^{(11)} \sim R_{11} G_{N}^{(10)} \sim M_{11}^{-9}$, the characteristic mass scale of the 11-dimensional theory is $M_{11}=g^{-1 / 3} l_{s}^{-1}$. At energies $\mathcal{O}\left(M_{11}\right)$, neither string theory nor supergravity are adequate descriptions. Both have to be superseded by an as yet unknown theory which has been given the name $M$-theory. The strongly coupled $E_{8} \times E_{8}$ heterotic string can also be interpreted as a compactification of M-theory, namely on an interval. The gauge degrees of freedom of one $E_{8}$-factor are located on each of the two ten-dimensional boundaries at the end of the interval.

The duality relations imply that the five string theories are merely different perturbative approximations of one and the same fundamental theory. The fact that 11-dimensional supergravity also appears indicates that the five string theories cannot provide a complete description in the strong coupling regime. The hypothetical theory, from which the five string theories and 11dimensional supergravity can be derived in different approximations, is called M-theory. The elementary excitations of this theory depend on the approximation. As 11-dimensional theory it possesses membranes, i.e. M2-branes, and their dual objects, M5-branes. The fundamental string of type IIA arises upon compactification on a circle of radius $R_{11}$ where the M2 branes is wrapped around the circle.

In addition to the duality relations which was we have discussed here, there are other connections between various string theories, in the critical dimension as well as in the compactified theory. In all non-perturbative dualities branes play an essential rôle.

In the presence of D-branes one has, besides the excitation modes of the closed string, also those of the open string whose endpoints move along the world-volume of the branes. For instance, at low energies $\left(l_{s} \rightarrow 0\right)$, the dynamics of the massless modes of $N$ coincident D3-branes is described by a four-dimensional $\mathcal{N}=4$ SYM-theory with gauge group $U(N)$. This gauge theory is localized on the world-volume of the D3-branes. Its gauge coupling constant is $g_{\mathrm{YM}}^{2}=g$. In the limit $l_{s} \rightarrow 0$ the modes of the open string and gravity decouple. Many different theories can be constructed by an appropriate choice of D-brane configurations and e.g. many features of the vacuum structure of the supersymmetric extension of QCD (SQSD) can be 'understood' in the brane picture.

\section{$7 \mathrm{AdS} / \mathrm{CFT}$}

String theory dates back to the pre-QCD era, as an attempt to understand the scattering data of hadrons. Veneziano 'guessed' a formula (known as the Veneziano formula) which correctly incorporates the empirically motivated duality hypothesis, which states that the complete four-point amplitude can be written either as a sum over only $s$-channel poles or as a sum over only $t$-channel poles. It was soon realized that the Veneziano amplitude can be derived from a theory of (bosonic) strings. Serious problems related to the 
high-energy behaviour of the Veneziano amplitude and, in particular, the discovery of QCD as a renormalizable QFT made string theory as a theory of the strong interaction obsolete. Furthermore, the discovery of a critical dimension and the presence of a massless spin-two particle were considered as indications that string theory might be the correct framework for a theory of quantum gravity. This has become the prevailing point of view.

However, more recently, based on the AdS/CFT conjecture of Maldacena, string theory has become a powerful analytic tool for studying strongly coupled gauge field theories. The most interesting such theory is QCD at low energies. While no gravity dual has yet been found, many (supersymmetric) generalizations have been studied using the so-called 'gauge theory - gravity duality'.

In its simplest version, the AdS/CFT correspondence arises from analysing a system of $N$ coincident D3 branes. For small $g N=g_{\mathrm{YM}}^{2} N$, i.e. for small 't Hooft coupling, the world-volume theory on the branes is the conformally invariant $U(N) \mathcal{N}=4$ supersymmetric gauge theory. Its degrees of freedom arise from the massless excitations of the open strings ending on the branes. This theory is coupled to supergravity in the ten-dimensional space-time. The supergravity fields arise from massless excitations on the closed strings. As long as $g N$ is small, one can neglect the backreaction of the branes on the geometry and the assumption of the D3 branes embedded in ten-dimensional Minkowski space-time is appropriate. In the limit $l_{s} \rightarrow 0$ the gauge theory on the brane decouples from the gravity theory in the bulk. If $g N$ becomes large, the backreaction can no longer be neglected and the system is better described by the geometry of the brane solutions of type IIB supergravity. The above decoupling limit now leads to a decoupling of the region close to the branes, the so-called 'near-horizon region' which has $A d S_{5} \times S^{5}$ geometry, from the asymptotic region, where one obtains a theory of free gravitons in ten-dimensional Minkowski space-time. Comparison then suggest a correspondence between four-dimensional $\mathcal{N}=4 \mathrm{SYM}$ theory and type IIB string theory compactified on $A d S_{5} \times S^{5}$. It also implies the relations $\left(R / l_{s}\right)^{4}=4 \pi g N=4 \pi g_{\mathrm{YM}}^{2} N$ between the string scale $l_{s}$, the curvature radius $R$ of the background geometry, the string coupling constant $g$, the rank of the gauge group $N$, and the gauge coupling constant $g_{\mathrm{YM}}^{2}$. One can think of the gauge theory degrees of freedom to be located at the conformal boundary of $A d S_{5}$ which is four-dimensional Minkowski space-time (up to global issues). In this sense, the AdS/CFT correpondence is a very concrete realization of the holographic principle (see also Sect. 8). One can further interpret the radial coordinate as the energy scale in the field theory.

As long as the radius of curvature is large and the string coupling constant is small, one can approximate the type IIB string theory by IIB supergravity on this background. One then obtains a duality between a quantum field theory $-\mathcal{N}=4 \mathrm{SYM}$ in the large- $N$ limit - and a classical gravity theory. Evidence for this duality is provided by a matching of the symmetries: the isometry group of the space-time coincides with the global symmetries of the 
gauge theory (conformal invariance and $\mathcal{R}$-symmetry) and this extends to the supergroups. In particular the $A d S$-factor of the geometry indicates that the dual field theory is conformally invariant (which $\mathcal{N}=4 \mathrm{SYM}$ is) 8 More detailed checks, which do not rely entirely on the symmetries, have been performed. For instance, the conformal anomaly of $\mathcal{N}=4 \mathrm{SYM}$, which is clearly a quantum effect of a four-dimensional field theory, can be computed via a classical gravity calculation. A precise matching between Kalazu-Klein states of the supergravity theory and gauge invariant operators is possible and many of their dynamical properties can be computed on both sides of the correspondence. Needless to say that they match precisely.

One obstacle to go beyond the supergravity approximation on the string theory side is that this requires the quantization of string theory compactified on $A d S_{5} \times S^{5}$, which consists, in addition to a background metric, of a non-vanishing value of the self-dual $(\mathrm{R}, \mathrm{R})$ five-form field strength. At present, quantization in (R,R) backgrounds (as opposed to (NS,NS) backgrounds) is still an unsolved problem, at least in the so-called 'RNS' (Ramond-NeveuSchwarz) formalism on which most of the string literature is based. But it has been shown that $A d S_{5} \times S^{5}$ is a consistent background for string compactification to all orders in string pertubation theory.

Considerable progress has been made in the so-called 'BMN' (BerensteinNastase-Maldacena) limit where relevant configurations on the string side are classical solutions of the string sigma-model which correspond to macroscopically large strings rotating in the background geometry. On the gauge theory side the dual operators are those with large conformal dimension and $R$-charge (which is dual to the $S O(6)$ isometry of $S^{5}$ ).

Many generalizations of the correspondence have been constructed. For instance, in order to reduce the amount of supersymmetry one replaces $S^{5}$ by a five-dimensional compact manifold $X^{5}$ which can serve as the base of a six-dimensional Ricci-flat Kähler cone, i.e. $X^{5}$ must be a Einstein-Sasaki manifold (e.g. for $X^{5}=S^{5}$ the Kähler cone is simply $\mathbb{R}^{6}$ ). Generalizations to non-conformal theories are neccessary if one wants a dual description of confining gauge theories (such as QCD). In fact, one can give a rather general criterium which the background geometry has to satisfy in order that the dual gauge theory is confining. This relies on the picture of the QCD string as a fundamental string which connects two quarks which are located on the 'boundary' of the dual geometry, but which plunges into the bulk (cf. Fig. 7) as this is the geodesic which connects its two endpoints. The expectation value of the Wilson loop $\langle W[\mathcal{C}]\rangle \sim e^{-T E(L)}$, where $E(L)$ is the potential energy, is the exponentiated area of the world-sheet of the open string which boundary $\mathcal{C}$, computed with the Nambu-Goto action in the given background geometry. If one does this in the $A d S_{5} \times S^{5}$ geometry one finds $E(L) \sim T / L$, i.e. the Coulomb law. In a confining dual geometry one finds instead $E(L)=\sigma T L$,

\footnotetext{
${ }^{8}$ More generally, asymptotic $A d S$ geometries are dual to field theories which are conformally invariant (fixed point of the beta-function) in the UV.
} 


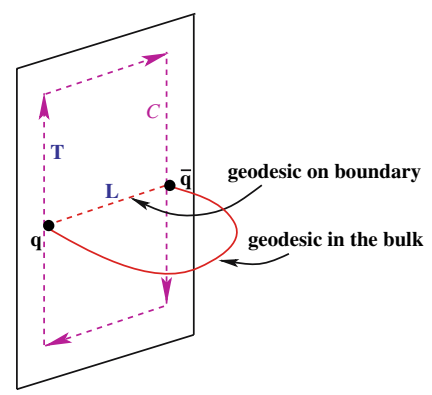

Fig. 7. 'QCD' string

where $\sigma$ is the QCD string tension. Such a confining geometry must contain an additional scale which breaks conformal invariance, in terms of which $\sigma$ can be expressed.

However, the regions in parameter space where, on the one hand, the gauge theory is weakly coupled and perturbation theory appropriate and, on the other hand, where the string coupling is weak and the space-time curvature small, i.e. where the supergravity approximation of string theory is good, do not overlap. Therefore, a direct comparison is only possible for protected operators which exists in field theories with extended supersymmetry (BPS states). But one can use the conjectured correpondence to arrive at 'predictions' about strongly coupled gauge theories, e.g. about their phase structure, the spectrum of mesons, chiral symmetry breaking, etc. in regimes where other analytical methods are not available. All these concepts have a geometric analogue within the dual gravity description. Also, the interpretation of the radial coordinate as the energy scale has been made precise, e.g., in extracting the gauge theory beta-function from geometrical data.

Other generalizations of the Maldacena conjecture lead to holographic descriptions of theories in other than four dimensions. For instance, compactification of 11-dimensional supergravity on $A d S_{7} \times S^{4}$ leads to a six-dimensional theory of interacting tensor multiplets on the world-volume of coincident M5branes. Again, the number of branes $N$ is related to the radius of curvature of the geometry $\left(R \sim l_{p} N^{1 / 3}\right)$. Nothing is known about this theory from the field theory side but definite predictions, e.g. about the number of degrees of freedom, i.e. that it grows as $N^{3}$, can be obtained from its dual gravity description.

Perhaps the most important lesson from these developments is the duality between quantum field theories (without gravity) and higher-dimensional gravitational theories (such as supergravity or string theory). A dual description of real QCD (four-dimensional, non-supersymmetric, $S U(3)$ gauge group, etc.) has not yet been found. But it has been demonstrated that the high energy behaviour of the Veneziano amplitude, when interpreted within AdS/CFT context (generalized to non-conformal backgrounds) where the 
radial coordinate serves as the energy scale, changes and that it is no longer in disagreement with experiments.

\section{Black-Hole Entropy}

Black holes are a major testing ground for ideas about quantum gravity. They are subject to the laws of black-hole mechanics which formally have the same structure as the laws of thermodynamics. Combining this with the Hawking effect, which allows to assign a temperature to a black hole, this leads to the identifications

$$
T=\frac{\kappa_{S}}{2 \pi}\left(\frac{\hbar}{c}\right), \quad \mathcal{S}_{\text {thermo }}=\frac{A}{4}\left(\frac{c^{3}}{G_{N} \hbar}\right) .
$$

Here $T$ is the Hawking temperature, $\mathcal{S}_{\text {thermo }}$ is the Bekenstein-Hawking entropy, $\kappa_{S}$ the surface gravity, and $A$ the area of the event horizon 9 The occurrence of $\hbar$ and $G_{N}$ clearly shows that black-hole entropy can only be described within the framework of a theory of quantum gravity. Below we will often use Planckian units and set $c=\hbar=G_{N}=1$. In analogy to the relation between thermodynamics and statistical mechanics, this suggests that while Einstein gravity describes black holes at the macroscopic level, a theory of quantum gravity should provide the microscopic description. In particular, it should be possible to relate the thermodynamical entropy to a statistical entropy, which measures the degeneracy of microscopic states for a given macroscopic state,

$$
\mathcal{S}_{\text {stat }}=\log N(M, Q, J) \text {. }
$$

Here the macroscopic state of a black hole is characterized by its mass $M$, charge $Q$, and angular momentum $J$, and $N(M, Q, J)$ denotes the number of microscopic black-hole states with given values for these quantities. It is a benchmark for any candidate theory of quantum gravity whether such microscopic states can be identified and counted, and whether the thermodynamical and statistical entropies agree.

The simple fact that the entropy is proportional to an area (the area of the black-hole's event horizon) rather than a volume, leads to the concept of holography. The information contained inside the region enclosed by the horizon is represented as a hologram on the horizon: all information about the inside is stored on the holographic screen 10 This is in sharp contrast with

${ }^{9}$ For a Schwarzschild black-hole $\kappa_{S}=c^{4} /\left(4 G_{N} M\right)$ and $A=4 \pi\left(\frac{2 M G_{N}}{c^{2}}\right)^{2}$.

10 More generally, the holographic principle asserts that the information contained in some region of space can be represented as a 'hologram' - a theory which 'lives' on the boundary of that region. It furthermore asserts that the theory on the boundary of the region of space in questions should contain at most one bit of information per Planck area $l_{\text {Planck }}^{2}$. 
what we expect from statistical mechanics and local quantum field theory where the entropy is an extensive quantity and should thus be proportional to the volume of the system. The lesson we learn from this is that the nature of the degrees of freedom of quantum gravity is quite different from that of a local quantum field theory.

In this section we discuss some of the research done on black-hole entropy in string theory. Except for the initial discussion of the heuristic string-black hole correspondence, we restrict ourselves to BPS black holes, i.e. black holes which are invariant under a subset of the supersymmetry transformations of the underlying string theory. For BPS black-holes, string theory provides a quantitative explanation of black-hole entropy. The agreement between thermodynamical and statistical entropy extends beyond the leading term in the semiclassical limit. Moreover, the calculations show that at subleading level the entropy of stringy black holes follows Wald's generalized formula for blackhole entropy, which deviates from the simple area law once quantum corrections (higher derivative corrections) to the Einstein-Hilbert action are taken into account.

We first discuss the heuristic string-black hole correspondence which, while qualitative, has the virtue to apply to Schwarzschild-type black holes. The basic idea is that 'heavy strings states are black holes'. Let us start with string perturbation theory in flat space. Free strings have an infinite tower of states of ever increasing mass, $m^{2} \sim \frac{n}{\alpha^{\prime}}, n \in \mathbb{N}$. If we take the string coupling $g$ to be finite, but small, the feedback of a sufficiently light string state on its ambient space-time is negligible. A rough way of estimating this feedback is to compare the characteristic length scale of string theory, $l_{S}=\sqrt{\alpha^{\prime}}$, to the gravitational scale of a string state of mass $m$, i.e. its Schwarzschild radius $r_{S} \sim G_{N} m \sim \sqrt{n \alpha^{\prime}} g^{2}$. Here we used the relation $G_{N} \sim g^{2} \alpha^{\prime}$ between the four-dimensional Newton constant $G_{N}$, the string scale $\sqrt{\alpha^{\prime}}$, and the string coupling constant, together with the mass formula 11 The string length $\sqrt{\alpha^{\prime}}$ is the smallest length scale which can be resolved by scattering string states. Since the feedback of a string state on the space-time geometry is estimated by $r_{S}$, it is negligible if $\sqrt{\alpha^{\prime}} \gg r_{S}$. For given coupling this requires that the mass of the state is sufficiently small, while for given mass the coupling must be sufficiently small. In this regime the number of string states of given mass can be counted, since we know the spectrum of free strings in flat space-time. The asymptotic number of states is governed by the Hardy-Ramanujan formula and grows like $e^{\sqrt{n}}$. In other words the statistical entropy of string states grows like

$$
\mathcal{S}_{\text {stat }} \sim \sqrt{n}
$$

for large $n$.

Let us now either increase the mass, at fixed coupling, or increase the coupling, at fixed mass. Then $r_{S}$ will grow relative to $\sqrt{\alpha^{\prime}}$. While we do not

${ }^{11}$ We assume that the additional dimensions required for consistency have been compactified on a manifold of size $\left(\alpha^{\prime}\right)^{3}$. 
know what happens in detail, we know qualitatively what happens in the opposite extreme regime $r_{S} \gg \sqrt{\alpha^{\prime}}$, where the gravitational scale is much larger than the string scale. Here we can use that the long-wavelength approximation of string theory is provided by an effective field theory, which can be constructed using string perturbation theory. The effective action has an expansion in string loops, controlled by $g$, so that we need to keep the string coupling small enough. Moreover, it has an expansion in the string length $\sqrt{\alpha^{\prime}}$. The long-wavelength or low energy expansion of the action is an expansion in derivatives. $\sigma$-model loop corrections (i.e. higher orders in $\alpha^{\prime}$ ) which appear in each order in the genus expansion (higher orders in $g$ ) give rise to higher derivative terms.

In this regime gravity is described by the Einstein-Hilbert action plus an infinite series of higher curvature terms. For the time being, we only take into account the leading Einstein-Hilbert term. Then we are in the realm of general relativity and expect that an object which sits within its Schwarzschild radius forms a black hole. Therefore our original string state should correspond to a black-hole solution of the effective field theory. The associated thermodynamical entropy is the Bekenstein-Hawking entropy. For a Schwarzschild-type black hole carrying the mass of the string state we obtain

$$
\mathcal{S}_{\text {thermo }}=\frac{A}{4 G_{N}} \simeq G_{N} m^{2} \simeq g^{2} n .
$$

Comparing this to the statistical entropy of string states (8.3), we see that both entropies are different, in general. However, they agree, up to a numerical constant of order unity, when the Schwarzschild radius is of the order of the string scale, $r_{S} \simeq \sqrt{\alpha^{\prime}}$, or, equivalently, for a string coupling of order $g^{2} \sqrt{n} \simeq 1$. The observation that the entropies of strings and black holes match here support the idea of a phase transition (or maybe a smooth crossover) from a perturbative string regime to a black-hole regime. In particular, this provides a scenario for the endpoint of the decay of black holes through Hawking radiation: once the black hole has shrunk to a size of order $\sqrt{\alpha^{\prime}}$, it converts into a highly excited string state, which then decays according to the rules of string perturbation theory. It is encouraging that a string has the right number of states to account for the states of a black hole with equal mass. This scenario is compatible with unitarity, and elaborates on the old idea of a correspondence between black holes and elementary particles. The idea of a phase transition is further supported by the observation that the Hawking temperature of a black hole of size $\sqrt{\alpha^{\prime}}$ equals the Hagedorn temperature, which is interpreted as the limiting temperature for a grand canonical ensemble of strings.

While this scenario is broad and appealing, it is very qualitative. In particular, the string and black-hole entropy only match up to a multiplicative factor of order unity, and the interpolation between the perturbative string regime and the black-hole regime is bold, because one has no control over the intermediate regime. There is no a priori argument which connects the number 
of states in the two extreme regimes, which in principle could change drastically. And indeed, we saw that the two entropies are different in general 12 indicating that the number of states changes when going from one extreme regime to the other.

Therefore we will now focus on a subset of states which are under much better control. Here, the entropies of strings and black holes will not just match for some particular value of the coupling, but they will be equal. Here 'equal' means equality up to additive terms which are subleading in the semiclassical limit, corresponding to large mass. In particular, there is no undetermined or mismatching factor between the leading terms of the two entropies. The relevant subclass of states are the BPS states, which sit in special, so-called 'short' or BPS representations of the supersymmetry algebra. BPS states carry central charges under the supersymmetry algebra, and have the minimal mass compatible with their central charges. In supergravity the central charges are determined by the electric and magnetic charges under gauge interactions which are mediated by the gauge bosons in the supergravity multiplet (graviphotons).

In string perturbation theory, BPS states appear as a special subset of the string states. In the corresponding effective supergravity theory, BPS states are realized as supersymmetric solitons, more specifically as extremal black-hole solutions with Killing spinors. The comparison of black hole and string entropy proceeds by constructing BPS black-hole solutions with given charges and by comparing the resulting entropy to the number of string BPS states with the same mass and the same charges. In various examples where both entropies have been computed in their respective regimes, it has been found that they agree, even when including subleading corrections.

Let us discuss an explicit example for the quantitative version of the stringblack hole correspondence. We consider four-dimensional string compactifications with $\mathcal{N}=4$ supersymmetry. For concreteness, we employ the realization through the heterotic string, compactified on a six-torus. For generic moduli the gauge group of this compactification is $U(1)^{28}$, and the electric charges carried by elementary string states can be combined into a vector $\mathbf{q}$ which takes values in a 28-dimensional lattice $\Gamma_{22,6}$, which comes equipped with an indefinite bilinear form of signature $(22,6)$. Incidentally, the problem of counting BPS states of charge $\mathbf{q}$ is equivalent to counting the number of states for the open bosonic string in 26 dimensions. Hence the result follows from the Hardy-Ramanujan formula. The corresponding entropy is

$$
\mathcal{S}_{\text {stat }}=4 \pi \sqrt{\frac{\left|\mathbf{q}^{2}\right|}{2}}+\cdots \text {. }
$$

Here $\mathbf{q}^{2}$ is the (indefinite) scalar product of the charge vector $\mathbf{q} \in \Gamma_{22,6}$ with itself. We have displayed the leading contribution in the limit of large charges

$\overline{12}$ Note that the black-hole entropy is bigger than the string entropy if we are in the black-hole regime, and vice versa. 
$\left|\mathbf{q}^{2}\right| \gg 1$ (which, through the BPS condition, implies large mass). There are corrections, starting with a term proportional to $\log |\mathbf{q}|^{2}$, followed by an infinite series of terms which involve negative powers of $\left|\mathbf{q}^{2}\right|$, plus further corrections which are exponentially suppressed for large charges.

The corresponding effective field theory is, to leading order in derivatives, an $\mathcal{N}=4$ supergravity theory coupled to 22 vector multiplets. It turns out that BPS solutions with charges $\mathbf{q}$ always have a null singularity, i.e. the event horizon coincides with the singularity and has vanishing area. As a consequence, the Bekenstein-Hawking entropy is zero

$$
\mathcal{S}_{\text {thermo }}=\frac{A}{4}=0,
$$

and disagrees with the statistical entropy of string states. This is, however, not the end of the story. Since space-time curvature becomes large close to the horizon, one cannot trust the two-derivative effective action. Once the leading curvature-squared terms are taken into account, the null singularity is replaced by a smooth horizon of area $A=8 \pi \sqrt{\frac{1}{2}\left|\mathbf{q}^{2}\right|}$. The corresponding BekensteinHawking entropy $\mathcal{S}_{\text {Bekenstein-Hawking }}=\frac{A}{4}=2 \pi \sqrt{\frac{1}{2}\left|\mathbf{q}^{2}\right|}$ is finite, but disagrees with the statistical entropy by a factor 2 . However, as pointed out some time ago by $\mathrm{R}$. Wald, the area law has to be replaced by a more refined formula, once the gravitational action contains higher derivative terms. In contrast to the naive area law, Wald's modified law assures that the first law of blackhole mechanics remains valid. For the case at hand, Wald's modified formula amounts to an additive correction term $\frac{A}{4}$, which leads to precise agreement between the leading term of the thermodynamical entropy

$$
\mathcal{S}_{\text {thermo }}=4 \pi \sqrt{\frac{\left|\mathbf{q}^{2}\right|}{2}}+\cdots
$$

and the statistical entropy. Like the statistical entropy, the thermodynamical entropy is further modified if subleading corrections are taken into account. For the thermodynamical entropy, the corrections come from further subleading terms in the effective action. As for the statistical entropy, these corrections are logarithms, inverse powers, and exponentials in $\left|\mathbf{q}^{2}\right|$.

The next step is therefore to compare the subleading contributions to both entropies. In the above example, no full agreement between statistical and thermodynamical entropy has been achieved to date. The problem seems to be related to the fact that for BPS black holes in $\mathcal{N}=4$ compactifications, which carry only electric charge, the scalar fields take values in a particular subspace of the moduli space, which is singular unless instanton corrections are taken into account. This reflects itself in the fact that black-hole solution has a vanishing horizon area at leading order. While further work is needed to better understand this class of BPS black holes, the situtation is much better for generic BPS black holes, which carry both electric and magnetic charges. 
The most general BPS black-hole solution of an $\mathcal{N}=4$ compactification carries 28 electric charges $\mathbf{q}$, but also 28 magnetic charges $\mathbf{p}$, which lie on a lattice of the form $\Gamma_{22,6} 13$ When using the two-derivative effective action, the entropy of such dyonic BPS black holes is

$$
\mathcal{S}_{\text {thermo }}=\pi \sqrt{\mathbf{p}^{2} \mathbf{q}^{2}-(\mathbf{p} \cdot \mathbf{q})^{2}} .
$$

Observe that for purely electric charge the entropy vanishes. This is the subcase we discussed above. What are the corresponding string theory microstates? Fundamental strings do not carry magnetic charges with respect to the gauge group $U(1)^{28}$. However, magnetic charges are carried by heterotic five-branes, which are solitonic objects occurring in the heterotic string theory. Dyonic BPS states with arbitrary electric and magnetic charge correspond to bound states of fundamental heterotic strings and heterotic five-branes. The number of BPS states with given charges is known in terms of an integral representation. When evaluating this integral at its leading saddle point, one recovers (8.6). But as in the case (8.4) there are subleading corrections to both the statistical and thermodynamical entropy. This time the corrections agree even when including contributions which are exponentially suppressed for large charges. At the level of the effective action, this corresponds to including the contribution of an infinite series of instanton corrections to the higher-derivative terms. The agreement crucially depends on using Wald's modified formula instead of the naive area law.

There are several other types of brane configurations where a quantitative agreement between statistical and thermodynamical entropy, including subleading corrections, has been found. In particular, the first examples of such a matching involved D-branes, rather than fundamental strings and solitonic five-branes. With this amount of evidence, it is fair to say that string theory can account quantitatively for the entropy of BPS black holes. String theory is unrivaled in that the matching of statistical and thermodynamical entropy does not involve the tuning of free parameters, and that the matching extends to subleading corrections and is sensitive to the distinction between Wald's law and the area law. This success also illustrates that a consistent perturbative theory of quantum gravity accounts for much more than 'graviton scattering in a fixed background'. In particular, string perturbation theory can be used to derive higher curvature corrections to the Einstein-Hilbert action. These in turn modify black-hole solutions, smooth singularities, and give contributions to the entropy. These are genuine quantum gravity effects, as the higher-derivative terms are generated by quantum corrections 14 While the

$\overline{13}$ By Dirac quantization, electric and magnetic charges lie on dual lattices. However, the charge lattice turns out to be self-dual, so that one has two copies of the same lattice.

${ }^{14}$ To be precise, the terms relevant for the $\mathcal{N}=4$ compactifications discussed above are 'tree-level plus instantons' (in the string coupling $g$ ) for the heterotic string and 'one-loop' for the dual description by the type-II string. 
agreement of statistical and thermodynamic entropy strongly suggests that string theory has the right number of degrees of freedom to account for the microstates of BPS black holes, a more direct understanding of these states as states of black holes is certainly needed. Recently, an intriguing proposal has been put forward by H. Ooguri, A. Strominger, and C. Vafa, which defines a 'black hole partition function' and relates it to the partition function of the topological string. This could be a major step forward in this direction.

A clear limitation of the approach described here is that it relies on supersymmetry, or, to be precise, that it applies to supersymmetric states only. However, there are other approaches to black holes within string theory, which we are not able to discuss here for lack of space. But let us mention that recently there has been considerable interest in studying non-supersymmetric extremal black holes. It turns out that many features of supersymmetric black holes carry over, and in particular that higher derivative corrections can be taken into account. Moreover, black-hole entropy has been studied extensively in the context of the AdS/CFT correspondence, which can be viewed as a concrete realization of the 'holographic principle' 15 Finally, a new line of thought is the 'fuzzball proposal', which views BPS black holes as superpositions of smooth geometries, one for each black-hole microstate. This approach might be a first step towards a detailed understanding of the interpolation between the string perturbative regime and the black-hole regime.

So far, string theory does not yet provide a complete account of black-hole physics. Nevertheless, black-holes are clearly the most successful application of string theory in the gravitational realm. They will continue to be a major subject of interest in the string community, and, maybe, the results will even reshape our understanding of what string theory is.

\section{Approaches to Phenomenology}

As we discussed in Sect. 5 the spectrum of excitations of a string compactified, e.g., on a Calabi-Yau manifold, contains a finite number of massless excitations $L$ and an infinite number of massive modes $H$. Their mass is of the order of the characteristic scale of the string $M_{s}$. Among the massless modes one finds generically a spin-2 degree of freedom which is identified with Einstein's graviton. In addition massless spin-1 gauge bosons of some gauge group $G$, families of massless chiral fermions in fundamental and anomaly free representations of $G$ and elementary spin-0 bosons which can serve as candidates for Higgs-like fields can appear among the massless modes. Such string backgrounds are not only a candidate for a consistent quantum gravity but also a candidate for a unified theory of all known particles and their interactions.

In order to check this proposal, it is necessary to identify the standard model (SM) as the low energy limit. This amounts to the identification of the

$\overline{15}$ The duality with a unitary quantum field theory strengthens the claim that there is no information loss during black-hole evaporation via Hawking radiation. 
particle spectrum of the standard model (or some generalization thereof) as well as their couplings in a low-energy effective Lagrangian $L_{\text {eff }}$. The effective Lagrangian can be computed systematically in perturbation theory by studying string scattering processes at energy scales $E$ far below the characteristic scale $M_{s}$. Demanding that the S-matrix of the effective field theory coincides with the string S-matrix for energy scales, $E \ll M_{s}$ determines the effective Lagrangian.

However, the programme just outlined has a number of serious drawbacks. First of all, the S-matrix elements in string theory can currently only be reliably computed as a perturbative expansion in the string coupling $g$. Second of all, a large class of consistent S-matrices, each corresponding to a twodimensional conformal field theory, do exist. This in turn leads to a large number of different effective theories with different $L_{\text {eff }}$. Every set of S-matrix elements (or equivalently every consistent CFT) can be viewed as a different vacuum of the same string theory. Each string vacuum is as good as any other or, in other words, the vacuum is degenerate and there is presently no understanding what selects one vacuum over another and lifts the vacuum degeneracy. Finally, string theory only contains one scale $M_{s}$ and one dimensionless coupling $g$ and hence all light modes $L$ are exactly massless. This is reminiscent of a standard model without the Higgs mechanism where all fermions and gauge bosons are also exactly massless. Thus, one has to understand what mechanism generates the weak scale $M_{Z}$ (and why it is so small).

Given this state of affairs there are a number of possible strategies to make further progress. One approach - commonly called 'string phenomenology' does not attempt to explain the mechanism which lifts the vacuum degeneracy and chooses the true vacuum. Rather it surveys the whole space of string ground states and looks for particularly 'promising' candidate vacua. The criteria of what is a 'promising' string vacuum is of course ambiguous and different aspects have dominated this field over the years. After the discovery of the heterotic string and its Calabi-Yau compactification in 1984/85 all of string phenomenology focused on vacua of the $E_{8} \times E_{8}$ heterotic string with four flat space-time dimensions with Minkowskian signature, a gauge group $G \subset E_{8} \times E_{8}$ which is big enough to contain the $S U(3) \times S U(2) \times$ $U(1)$ of the SM and at least three light chiral generations. In addition, $\mathcal{N}=$ 1 local space-time supersymmetry was imposed at $M_{s}$ since it seems very difficult to understand how the hierarchy $M_{Z} / M_{s}$ can be generated and kept stable without supersymmetry 16 Furthermore, most of the known consistent string vacua with space-time fermions are already supersymmetric and within our current understanding supersymmetry appears to be a plausible (if not necessary) symmetry of string theory.

$\overline{{ }^{16} \mathcal{N}=1}$ is chosen since such supersymmetric theories can easily have chiral fermions. This is not possible for $\mathcal{N}>1$. 
Almost all string vacua contain gauge neutral scalar fields $M^{i}$ ('moduli of the compactification') which are flat directions of the perturbative effective potential. Thus their vacuum expectation values (VEVs) are undetermined in perturbation theory and therefore they are additional free parameters of a given string vacuum. They set the (inverse) gauge couplings $g_{\mathrm{YM}}^{-2}$ and the Yukawa couplings $Y$ of the theory. Thus, as in any QFT, both the gauge couplings and the Yukawa couplings are free parameters of the effective lowenergy string theory. However, the situation here is slightly better than in a QFT. First of all, the fact that the couplings depend on scalar field VEVs opens up the possibility of a dynamical determination of the couplings. If we understood what mechanism lifts the flat directions and induces a $\left\langle M^{i}\right\rangle$ we would have a dynamical way of understanding the values of the dimensionless couplings $g_{\mathrm{YM}}$ and $Y$. Furthermore, it is quite possible that for a given vacuum the ratio of Yukawa couplings displays some special properties which can be tested experimentally.

The perturbative heterotic string has the additional feature that the gauge coupling is universal at the tree level. The generic gauge group is a product of simple factors $G=\prod_{a} G_{a}$ with gauge couplings $g_{a}$ for each factor $G_{a}$ which are identical even without the existence of a covering GUT group

$$
g_{a}^{-2}=\operatorname{Re}\langle\Phi\rangle \quad \text { for all } a,
$$

where $\Phi$ is the dilaton field. (Strictly speaking there is an integer normalization factor $k_{a}$ in (9.1) which we have omitted here for simplicity.) Thus the perturbative heterotic string very generically predicts a universal gauge coupling. It also predicts the scale at which the coupling constants unify to be $M_{s} \approx 5 \cdot 10^{17} \mathrm{GeV}$. Current electro-weak precision data seem to favour a unification of the gauge couplings at approximately $3 \cdot 10^{16} \mathrm{GeV}$ which is indeed remarkably close to the string value. However, given the present precision the mismatch of a factor of 20 cannot be simply ignored.

Despite some of the successes of the heterotic string vacua there are a number of questions left unanswered in the perturbative approach outlined above. We still have to understand how the light modes get their masses, how $M_{Z}$ and the hierarchy is generated, what lifts the vacuum degeneracy and induces VEVs for the $M^{i}$, and finally how supersymmetry is broken at low energies. The belief (and hope) is that all of these problems are just an artefact of string perturbation theory and that once we understand the non-perturbative phase of string theory these problems will have a (hopefully realistic) solution.

Since we lack a fully developed non-perturbative formulation of string theory there are various ways to argue the structure of possible non-perturbative corrections. First, one might assume that the dominant non-perturbative effects arise at energy scales well below $M_{s}$ and therefore can be described by field-theoretic means. Clearly these non-perturbative effects are part of string theory and the real assumption is that they dominate over the 'stringy' effects. This assumption is partly motivated by the fact that in order to generate a 
hierarchy these non-perturbative effects have to occur at an energy scale well below $M_{s}$. An example of such a non-perturbative effect is gaugino condensation in a hidden sector which can be analysed already in supergravity. The hidden sector which has no renormalizable interactions with the observable sector is taken to be an asymptotically free non-abelian gauge theory which is weakly coupled at $M_{s}$ but becomes strongly coupled at

$$
\Lambda_{c}=M_{\mathrm{Pl}} e^{-\frac{8 \pi^{2}}{b g_{\mathrm{YM}}^{2}}} \ll M_{\mathrm{Pl}},
$$

where $b$ is the coefficient of the one-loop $\beta$-function. Such hidden sectors do indeed exist in string theory, the matterless $E_{8}$ of Calabi-Yau compactification of the heterotic string is only one example. As we already discussed, the gauge couplings are field dependent in string theory and thus a non-trivial potential for $M^{i}$ is generated

$$
g_{\mathrm{YM}}^{-2}\left(M^{i}\right) \rightarrow \Lambda_{c}\left(M^{i}\right) \rightarrow V_{\mathrm{np}}\left(M^{i}\right) .
$$

At the minimum of $V$ supersymmetry can be spontaneously broken and non-trivial VEVs for $M^{i}$ can be generated. However, generically a large cosmological constant arises in almost all of the models considered so far and no realistic scenario satisfying all phenomenological constraints has been constructed. As a consequence a more detailed low-energy phenomenology of such models has not been developed.

In recent years a slight variation on this setup has been studied which goes under the name of 'Brane World Scenarios'. Here the standard model or its generalization lives on a stack of space-time filling D-branes in a type II bulk. Supersymmetry is spontaneously broken by additionally turning on background fluxes in the bulk already at the tree level. The fluxes generate a potential which fixes some of the moduli but in general additional nonperturbative effects have to be employed in order to fix all of them and to obtain a (meta-stable) ground state. This aspect is particularly important if one attempts to construct de Sitter vacua with a small cosmological constant. A detailed analysis of these 'Brane World Scenarios' is currently under way.

If the gauge degrees of freedom of the standard model arise as excitations of a D-brane they can be viewed as localized on a three-dimensional plane within a higher-dimensional space. This implies that the 'extra' dimensions can only be probed by the gravitational interaction. Currently Newtons $\frac{1}{r}$ law is experimentally established down to the sub-millimeter range while the Coulomb $\frac{1}{r}$ law has been established in Bhabha-scattering at LEP down to $10^{-18} \mathrm{~m}$. This opens up the theoretical possibility of 'large extra dimension' which are only transparent for gravity. If they are large enough they can be seen as deviations from Newtons $\frac{1}{r}$ law in gravitational torsion experiments or at LHC by producing appropriate Kaluza-Klein excitations. The phenomenological signatures of such scenarios have been studied in detail. 


\section{Open Questions}

The most obvious shortcoming of 'perturbative string theory' is that it is limited to a set of rules for computing on-shell scattering amplitudes in an on-shell background. If we want to address conceptual issues of quantum gravity this is a double handicap: quantities can only be computed as formal power series in the string coupling, and one has to fix an on-shell background in advance. Perturbative and non-perturbative dualities have certainly enhanced the range of quantities which can be computed, but without changing these points fundamentally. Direct non-perturbative methods, such as an instanton calculus, are in a very early state of development. One might hope that string field theory supersedes the (conceptually) cumbersome 'first quantized' formalism which is still mostly used. However, string field theory is very complicated to work with. With the notable exception of tachyon condensation, string field theory has mainly been used to reproduce results obtained before in the 'first quantized' approach.

Manifest background independence is certainly a desirable feature of any theory of gravity. String theory is background independent, in the sense that different on-shell backgrounds are different solutions of one underlying theory. Formally, this is clear from the fact that deformations of on-shell backgrounds correspond to marginal deformations of the world-sheet action, which in turn are equivalent to inserting the vertex operator for a coherent string state into correlators. However, background independence is not manifest, as one needs to fix a reference background, or equivalently a world-sheet conformal theory before being able to deform it. Therefore there is always an, albeit conventional, cut between the space-time geometry (plus other background fields) and the dynamics in the background. Compared to approaches to quantum gravity which focus on quantizing four-dimensional Einstein gravity, string theory faces additional challenges. The various perturbative and non-perturbative dualities clearly indicate that there is a huge redundancy between the consistent string backgrounds. In particular, since dualities mix the gravitational with other degrees of freedom, one gets identifications between space-time geometries with different topologies. The most prominent example of this is mirror symmetry, which relates Calabi-Yau threefolds with opposite Euler numbers (and reflected Hodge diamonds), and 'second quantized mirror symmetry', which relates type-II string theory on certain Calabi-Yau threefolds to the heterotic string on $K 3 \times T^{2}$ (together with a certain choice of gauge fields inside the $K 3$-surface). While this appears to be a deep observation, what is lacking so far is a sufficiently abstract and general concept of 'state', which allows one to understand why these apparently different spacetimes (amended with other background fields) represent the same state. One closely related question is, what is the geometry underlying string theory?

Both quantum corrections, controlled by $g$ and stringy corrections, controlled by $\alpha^{\prime}$, have consequences for space-time geometry. Since dualities can exchange quantum effects and stringy effects, both kinds of modifications are 
related, and the distinction between them depends on the 'duality frame' one is using. So far, Calabi-Yau compactifications, in particular in the setting of the topological string, have been the major playground for exploring 'string geometry'. More work needs to be done in this framework before addressing these questions within the full theory. Note that topological string theory is rich enough to address issues such as background independence, quantum space-time structure ('space-time foam'), and the quest for a non-perturbative formulation.

The problem of 'string geometry' can also be rephrased from another perspective, by highlighting the distinction between 'geometrical backgrounds' and 'observed geometry'. Geometrical backgrounds are classical data, which are used to define the world-sheet conformal field theory, while observed geometry is the geometry one infers by probing space with string or brane states. This is illustrated by the example of strings in Minkowski space-time. While Minkowski space-time does not have a minimal length scale, the shortest length resolved by scattering string states is the string length $\sqrt{\alpha^{\prime}}$. Thus there is a qualitative difference between the classical background used to define the world-sheet theory, and the geometry 'seen' by strings. Things become more complicated if we probe the same geometry using different objects. In particular, D-particles (D0-branes) resolve a different minimal length scale, the 11-dimensional Planck scale, which is related to the string length scale through the vacuum expectation value of the dilaton. This again illustrates the high redundancy in the description of observable quantities. What is needed here is a disentanglement between observables and gauge symmetries. While all this is 'well known' within the string community, and has been discussed in several publications, a more focused effort might be needed to make progress in these important conceptual questions.

The simplest consistent string backgrounds are ten-dimensional Minkowski space, populated by either of the five supersymmetric perturbative string theories, and 11-dimensional Minkowski space, for which only the massless sector and the BPS states are known. This clearly presses the question why we live in a four-dimensional universe. Moreover, even when taking the attitude to impose that the additional space dimensions are unobservable at the presently realizable energy scales, one still meets the problem that there is a huge number of ways to compactify the theory to four dimensions. This is, first of all, a serious obstacle for testing the theory empirically based on its predictions. And, second, it leaves us with the question whether the particular solution which describes our universe (assuming that such a solution really exists) has been chosen by a historical accident, or whether there is a dynamical explanation. Since currently no convincing dynamical explanation is at hand, an eloquent group within the string community advocates the use of the anthropic principle within the context of eternal inflation. Not surprisingly, this move has provoked harsh criticism, which in its most pointed form discards anthropic reasoning as being unscientific. Before commenting on the anthropic principle, let us point out that it is not clear a priori which properties of our 
universe can be explained by recourse to laws and which properties are just historical facts. This is, of course, closely related to the distinction between 'equations' and 'initial conditions' which is the key point of a famous essay by E. Wigner. While for most branches of physics it is not controversial that science explains 'regularities among events', while initial conditions are contingent (true but not necessarily true), we nowadays tend to expect more than this of quantum cosmology. However, the idea that a theory could dispense itself from initial conditions, or could, in some sense, explain them, might just be wrong. This said, we need to stress that there are alternatives to anthropic considerations, which are worth exploring. As a matter of fact, the present state of string theory does not allow us to study generic timedependent space-times in the full theory. Therefore the main problem with anthropic reasoning is that it could prevent us from further developing the theory. A moderate goal, which has been subject of some recent activity, is to use effective fields theories, which incorporate some relevant stringy features, to show that string vacua with rich spectra of light (compared to string or Planck scale), stable, charged particles are preferred dynamically. Another, more demanding question is why four large space-time dimensions should be preferred. Ultimately, one needs to develop the formalism of string theory beyond the framework of fixed background on-shell amplitudes before these questions can be addressed properly.

\section{Some Concluding Remarks}

String theory has to a large extent been developed by exploring internal requirements of consistency, often in a formal rather than mathematically rigorous way. The underlying mathematical structure is very rich, and has lead to very non-trivial predictions, insights, and developments, which in turn have stimulated work by pure mathematicians and a vivid exchange of ideas between physicists and mathematicians. Some observations made in string theory, such as mirror symmetry, have already been put on firm ground. Topological string theory, which is not only a toy version of string theory but also a tool which allows to compute various quantities relevant for particle phenomenology, is well understood perturbatively, while a non-perturbative formulation is currently in the center of interest and might be within reach. This supports the expectation that a mathematically satisfactory formulation of the full string theory will be found eventually, although it is hard to estimate how long this will take. While there are good indications that the theory is consistent, its relevance for physics is less clear. Certainly, many ideas which have grown out of string theory, notably the AdS/CFT correspondence and the idea of extra dimensions, have had considerable influence on quantum field theory, particle physics, and gravitational physics. While some of these ideas are purely technical, like methods for the computation of amplitudes which are now commonly used in QCD (helicity amplitudes), other ideas are conceptual. 
In particular, string theory makes it very natural to express physical phenomena, including non-perturbative quantum phenomena in terms of geometry. Two prominent examples are the geometrical realization of strong-weak coupling dualities, as in the Seiberg-Witten solution of $\mathcal{N}=2$ gauge theories and its lifts to type-II string theory and 11-dimensional M-theory, and the holographic renormalization group, where the energy scale of a four-dimensional quantum field theory is literally treated as an extra dimension. But without direct empirical evidence, string theory might just be a technical tool, or a catalyser for ideas which one could also have developed independently. While this would not necessarily be bad in the sense of invalidating the work done in this field, most people working on string theory do certainly hope that it captures fundamental features of space, time, and matter. Then, finding ways of testing the theory through experiment or observation is indispensable.

From the perspective of the standard model one is eagerly waiting for experimental signatures which lead us beyond its domain of validity and, hopefully, indicate a particular type of extension. Whether signals of new physics will give us clues about the relevance of string theory will strongly depend on what kind of new physics will be found. Low-energy supersymmetry, with a rich spectrum of supersymmetric particles, would certainly be very attractive for particle phenomenology. It would also fit with the idea that physics at higher-energy scales is organized by higher symmetries, and would thus indirectly support string theory as the ultimate form of unified theory. However, it would also indicate that string effects only become relevant at the (four-dimensional) Planck scale, and then it will be very difficult to distill direct evidence for string theory out of the data.

The situation would be much better in the alternative scenarios with 'large' (TeV-scale rather than Planck scale) extra dimensions. This would involve gravity and it would also take us beyond the realm of renormalizable QFTs. In this case new concepts, such as those offered by string theory, become relevant already at the $\mathrm{TeV}$ scale.

Considerations of string theory have led to the discovery of very nontrivial mathematical structures, which might hold key for formulating a unified quantum theory of all interactions. This also gives confidence that there is a mathematically consistent and physically relevant theory underlying all the facets of what we today mean by string theory, whose complete fundamental structure and symmetries are still to be uncovered.

\section{Selected References}

Almost all papers on string theory since 1991 are available on http://de.arxiv. org/archive/hep-th. Below we will only give a few references which will guide the reader to more specific publications. 


\section{Popular Accounts}

B. Greene, "The Elegant Universe: Superstring, Hidden Dimensions, and the Quest for the Ultimate Theory," W. W. Norton \& Company, 1999.

L. Randall, "Warped Passages: Unraveling the Mysteries of the Universe's Hidden Dimensions," HarperCollins Publishers, 2005.

L. Susskind, "The Cosmic Landscape: String Theory and the Illusion of Intelligent Design," Little Brown and Company, 2005.

\section{Textbooks}

M. B. Green, J. H. Schwarz and E. Witten, "Superstring Theory. Vol. 1: Introduction, Vol. 2: Loop Amplitudes, Anomalies And Phenomenology," Cambridge University Press 1987.

D. Lüst and S. Theisen "Lectures on String Theory", Lect. Notes Phys. 346 (1989).

J. Polchinski, "String theory. Vol. 1: An introduction to the bosonic string, Vol. 2: Superstring theory and beyond," Cambridge University Press 1998.

B. Zwiebach, "A first course in string theory," Cambridge University Press 2004.

L. Susskind and J. Lindesay, "An Introduction to Black Holes, Information and the String Theory Revolution: The Holographic Universe," World Scientific Publishing 2004.

\section{Review Articles}

T. Mohaupt, "Black hole entropy, special geometry and strings,"

Fortsch. Phys. 49 (2001) 3, hep-th/0007195.

B. Pioline, "Lectures on on Black Holes, Topological Strings and Quantum Attractors,"

hep-th/0607227.

O. Aharony, S. S. Gubser, J. M. Maldacena, H. Ooguri and Y. Oz, "Large N field theories, string theory and gravity," Phys. Rept. 323, 183 (2000), hep-th/9905111.

A. Sen, "An introduction to non-perturbative string theory," hep-th/9802051. M. Grana, "Flux compactifications in string theory: A comprehensive review," Phys. Rept. 423, 91 (2006), hep-th/0509003 .

R. Bousso, "The holographic principle," Rev. Mod. Phys. 74, 825 (2002), hep-th/0203101

C. P. Bachas, "Lectures on D-branes," hep-th/9806199.

J. M. Maldacena, "Black Holes in String Theory," hep-th/9607235.

G. Horowitz, "Spacetime in string theory," New J. Phys. 7 (2005) 198, gr-qc/0410049. 